\title{
Stage IIIA Thymic Epithelial Neoplasm AJCC v8
}

National Cancer Institute

\section{Source}

National Cancer Institute. Stage IIIA Thymic Epithelial Neoplasm A/CC v8. NCI Thesaurus. Code C136327.

Stage IIIA includes: T3, NO, M0. T3: Tumor with direct invasion into any of the following: lung, brachiocephalic vein, superior vena cava, phrenic nerve, chest wall, or extrapericardial pulmonary artery or veins. NO: No regional lymph node metastasis. M0: No distant metastasis. (AJCC 8th ed.) 